\title{
Finding eigenvalues for matrices acting on subspaces
}

\author{
Jakeniah Christiansen * \\ Department of Mathematics and Statistics \\ Calvin College \\ Grand Rapids, MI 49546 \\ Faculty advisor: Prof. Todd Kapitula ${ }^{\dagger}$ \\ Department of Mathematics and Statistics \\ Calvin College \\ Grand Rapids, MI 49546
}

June 29, 2012

\begin{abstract}
Consider the eigenvalue problem $Q A Q \vec{x}=\lambda \vec{x}$, where $A$ is an $n \times n$ matrix and $Q$ is projection matrix onto a subspace $S^{\perp}$ of dimension $n-k$. In this paper we construct a meromorphic function whose zeros correspond to the (generically) $n-k$ nonzero eigenvalues of $Q A Q$. The construction of this function requires only that we know $A$ and a basis for $S$, the orthogonal complement of $S^{\perp}$. The formulation of the function is assisted through the use of the Frobenius inner product; furthermore, this inner product allows us to directly compute the eigenvalue when $k=1$ and $n=2$. When $n=3$ and $k=1$ we carefully study four canonical cases, as well as one more general case.
\end{abstract}

\section{Contents}

1. Introduction

3. The eigenvalue problem: $n=2$

4. The eigenvalue problem: $n=3$

4.1. Reformulation of the eigenvalue problem for $n \geq 3$. . . . . . . . . . . . . . . . . . . 194

4.2. Case study: $n=3$. . . . . . . . . . . . . . . . . . . . 196

4.2.1. Case 1: $A=D=\left(\begin{array}{ccc}\lambda_{1} & 0 & 0 \\ 0 & \lambda_{2} & 0 \\ 0 & 0 & \lambda_{3}\end{array}\right) \ldots \ldots \ldots \ldots \ldots$

4.2.2. Case $2: A=D=\left(\begin{array}{ccc}\lambda_{1} & 1 & 0 \\ 0 & \lambda_{1} & 0 \\ 0 & 0 & \lambda_{2}\end{array}\right)$

4.2.3. Case 3: $A=D=\left(\begin{array}{ccc}\lambda_{1} & 1 & 0 \\ 0 & \lambda_{1} & 1 \\ 0 & 0 & \lambda_{1}\end{array}\right)$

*E-mail: jcc7@students.calvin.edu

${ }^{\dagger}$ E-mail: tmk5@calvin.edu 


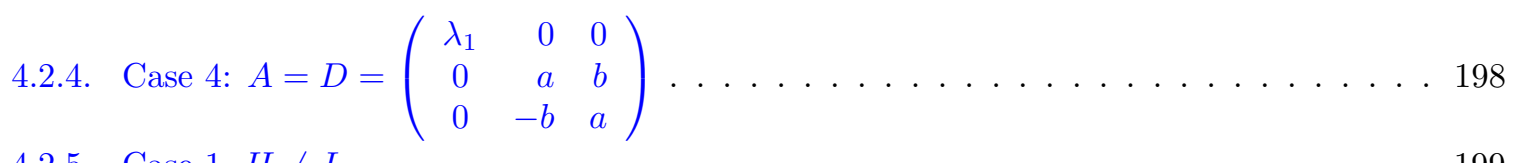

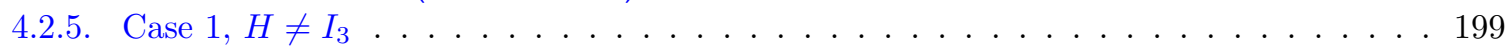

5. Conclusion $r$

$\begin{array}{ll}\text { References } & \mathbf{2 0 3}\end{array}$ 


\section{INTRODUCTION}

Consider the eigenvalue problem

$$
A \vec{x}=\lambda \vec{x},
$$

where $A$ is an $n \times n$ matrix with real-valued coefficients. From one physical perspective, the eigenvalue problem arises when considering the stability of critical points for Hamiltonian systems: in this case $A$ is symmetric, so that all of the eigenvalues are real-valued. In this context, if all of the eigenvalues are positive, then the critical point is energetically stable (i.e., the critical point is a local minimum of the energy surface) and is a stable (local) minimizer for the Hamiltonian flow. If some of the eigenvalues are negative, then it is expected that since the critical point is energetically unstable (i.e., the critical point is now a saddle point of the energy surface), then the critical point is actually unstable for the Hamiltonian flow. As it turns out, it is a little more complicated than that, as Hamiltonian flow on the energy surface may still allow the critical point to still be stable relative to the flow (e.g., see $[\mathbf{3}, \mathbf{6}-\mathbf{9}]$ for more details). It is important to find the sign of the eigenvalues.

Now suppose that the Hamiltonian system has $k$ conserved quantities associated with it (e.g., linear momentum, angular momentum, etc.), and for the moment further suppose that the critical point is a saddle point. Is the critical point really still energetically unstable? For example, it may be the case that if a solution were to travel along the energy surface in one of the unstable directions, then one or more of the conserved quantities would no longer be conserved. Since this is impossible, the presence of the conserved quantities does not allow a solution to access that particular unstable direction, which means that particular unstable direction on the energy surface does not really count as far as the flow is concerned. On the other hand, it may be the case that no conservation law is violated by a trajectory moving along an unstable direction. In order to understand the manner in which these conserved quantities affect the Hamiltonian flow near the critical point, one must then study a different, but related, eigenvalue problem, which is related to a Lagrange multiplier problem. This new eigenvalue problem will have associated with it a matrix which is related to $A$ of dimension $(n-k) \times(n-k)$, and the eigenvalues for this new eigenvalue problem will give information regarding the nature of the Hamiltonian flow in the presence of the conserved quantities. If for the new eigenvalue problem all of the eigenvalues are positive, then the critical point is stable in the presence of the conserved quantities. If some of the eigenvalues are negative, then it is still the case that the critical point is a saddle point, and hence (possibly) unstable.

The mathematical formulation of this new eigenvalue problem is a specific example of the following more general problem. Let $S$ be a real subspace of dimension $k$. Let $P$ represent the (orthogonal) projection matrix onto $S$, and let $Q=I_{n}-P$ be the projection matrix onto $S^{\perp}$, the subspace perpendicular to $S\left(\mathbb{R}^{n}=S \oplus S^{\perp}\right)$. Here $I_{n}$ is the $n \times n$ identity matrix. The eigenvalue problem to be studied in this paper is given by

$$
Q A Q \vec{x}=\lambda \vec{x}, \quad \vec{x} \in S^{\perp} .
$$

Since $Q A Q: S^{\perp} \mapsto S^{\perp}$ and $\operatorname{dim}\left(S^{\perp}\right)=n-k$, this eigenvalue problem is equivalent to an induced problem (with $\widetilde{A}=Q A Q$ )

$$
\widetilde{A} \vec{y}=\lambda \vec{y}, \quad \vec{y} \in \mathbb{R}^{n-k}
$$

(e.g., see [4]). The eigenvalues for (1.2) (or equivalently, (1.1)) can then be found by finding the zeros of the $(n-k)^{\text {th }}$-order characteristic polynomial $p_{\widetilde{A}}(\lambda)$, where

$$
p_{\widetilde{A}}(\lambda)=\operatorname{det}\left(\widetilde{A}-\lambda I_{n-k}\right) .
$$

In addition to the context provided above, eigenvalue problems of the form (1.1) also arise when looking for the so-called "Ritz values" of the matrix $A$ (e.g., see the discussion in [1, Chapter IV.2.2]). The question to be considered in this paper: is it necessary to explicitly compute $\widetilde{A}$ in order to find the $n-k$ zeros of $p_{\widetilde{A}}(\lambda)$ ?

An inverse form of this question was recently considered by Malamud [10] (also see the references therein). He considered the following problem: given a sequence of $n$ eigenvalues $\lambda_{j}$ and another sequence of $n-1$ eigenvalues $\mu_{j}$, does there exist a matrix $A$ and a projection $Q$ such that the $\lambda_{j}$ 's are the eigenvalues for $A$ and the $\mu_{j}$ 's are the eigenvalues for $Q A Q$ ? He showed that under certain conditions between the two sequences that there is indeed a unique quasi-normal Jacobi matrix $A$ and a subspace $S$ for which this is true [10, Theorem 3.7]. This result is a generalization for what was known for symmetric matrices [5]. 
In this paper we will consider the eigenvalue problem (1.1) without going through the process of computing the induced matrix $\widetilde{A}$. Since we then cannot compute $p_{\widetilde{A}}(\lambda)$, we must determine other ways and construct different functions to find the eigenvalues. In Section 2 we define the Frobenius inner product. In Section 3 we use the Frobenius inner product to directly solve (1.1) when $k=1$ and $n=2$. In Section 4 we solve the eigenvalue problem by first constructing a meromorphic function, say $E(\lambda)$, which has the property that its zeros correspond to eigenvalues. The construction of $E(\lambda)$ does not require us to directly compute $\widetilde{A}$; instead, all that is necessary is $A$ and a basis for $S$. We then use $E(\lambda)$ to study the eigenvalue problem in detail when $k=1$ and $n=3$. We close in Section 5 with some conclusions. The Appendix gives the details associated with some of the calculations presented in Section 4.

Acknowledgments. This work was supported in part by the National Science Foundation under grant number DMS-0806636. Special thanks to Professor Todd Kapitula for his guidance throughout this research project.

\section{The Frobenius inner Product}

The Frobenius inner product is defined as the component-wise multiplication of two matrices with realvalued coefficients, calculated as if finding the dot product of vectors:

$$
\langle A, B\rangle=\sum_{i} \sum_{j} A_{i j} B_{i j}
$$

for example,

$$
\left\langle\left(\begin{array}{ll}
a & b \\
c & d
\end{array}\right),\left(\begin{array}{ll}
e & f \\
g & h
\end{array}\right)\right\rangle=a e+b f+c g+d h .
$$

We may also note that $\langle A, B\rangle$ is equal to the trace of $A B^{\mathrm{T}}$. With this notion we have a different way of calculating inner products where matrices are involved, an original result based upon our research:

Theorem 2.1. For any $n \times n$ matrix $B$ and $n$-dimensional vector $\vec{s}, B \vec{s} \cdot \vec{s}=\left\langle B, \vec{s} \vec{s}^{T}\right\rangle$.

Proof: For

$$
B=\left(\begin{array}{ccc}
B_{11} & \ldots & B_{1 n} \\
\vdots & & \vdots \\
B_{n 1} & \ldots & B_{n n}
\end{array}\right)
$$

we have

$$
B \vec{s}=\left(\begin{array}{c}
B_{11} s_{1}+B_{12} s_{2}+\ldots+B_{1 n} s_{n} \\
B_{21} s_{1}+B_{22} s_{2}+\ldots+B_{2 n} s_{n} \\
\vdots \\
B_{(n-1) 1} s_{1}+B_{(n-1) 2} s_{2}+\ldots+B_{(n-1) n} s_{n} \\
B_{n 1} s_{1}+B_{n 2} s_{2}+\ldots+B_{n n} s_{n}
\end{array}\right) .
$$

Consequently,

$$
\begin{aligned}
B \vec{s} \cdot \vec{s}= & \left(B_{11} s_{1}+B_{12} s_{2}+\ldots+B_{1 n} s_{n}\right) s_{1}+ \\
& \left(B_{21} s_{1}+B_{22} s_{2}+\ldots+B_{2 n} s_{n}\right) s_{2}+\ldots \\
& \left(B_{(n-1) 1} s_{1}+B_{(n-1) 2} s_{2}+\ldots+B_{(n-1) n} s_{n}\right) s_{n-1}+ \\
& \left(B_{n 1} s_{1}+B_{n 2} s_{2}+\ldots+B_{n n} s_{n}\right) s_{n} .
\end{aligned}
$$

In other words, using the definition of the Frobenius inner product gives

$$
B \vec{s} \cdot \vec{s}=\left\langle B,\left(\begin{array}{cccc}
s_{1} s_{1} & s_{1} s_{2} & \ldots & s_{1} s_{n} \\
s_{2} s_{1} & s_{2} s_{2} & \ldots & s_{2} s_{n} \\
\vdots & & & \vdots \\
s_{n} s_{1} & s_{n} s_{2} & \ldots & s_{n} s_{n}
\end{array}\right)\right\rangle=\left\langle B, \vec{s} \vec{s}^{\mathrm{T}}\right\rangle
$$


Remark 2.2. Although we will not prove it here, it can be shown that in general

$$
A \vec{x} \cdot \vec{y}=\left\langle A, \vec{y} \vec{x}^{\mathrm{T}}\right\rangle
$$

\section{The eigenvalue PRoBlem: $n=2$}

Before studying the eigenvalue problem (1.1) in detail when $n=2$, we need the following preliminary original results discovered during our research, which are valid for any $n$ :

Corollary 3.1. For any $n \times n$ matrix $B$ and vector $\vec{v}$, for $C=\vec{v} \vec{v}^{\mathrm{T}}$ the solution to $C B C=\alpha C$ is given by $\alpha=\angle B, C\rangle$.

Proof: The form of $C$ yields that

$$
C B C=\vec{v}\left(\vec{v}^{\mathrm{T}}\right) B \vec{v}\left(\vec{v}^{\mathrm{T}}\right)=\vec{v}\left(\vec{v}^{\mathrm{T}} B \vec{v}\right) \vec{v}^{\mathrm{T}}=\vec{v}(B \vec{v} \cdot \vec{v}) \vec{v}^{\mathrm{T}} .
$$

Since by Theorem $2.1 B \vec{v} \cdot \vec{v}=\langle B, C\rangle$, we then note that

$$
C B C=\vec{v}\langle B, C\rangle \vec{v}^{T} .
$$

Since $\langle B, C\rangle$ is a constant this in turn implies that

$$
C B C=\langle B, C\rangle \vec{v} \vec{v}^{\mathrm{T}}=\langle B, C\rangle C,
$$

which is the desired result.

Corollary 3.1 can be used to solve the eigenvalue problem (1.1) (or equivalently (1.2)) in the case that $\operatorname{dim}\left(S^{\perp}\right)=1$. In this case there will exist a unit vector $s^{\perp}$ such that $Q=\vec{s}^{\perp}\left(\vec{s}^{\perp}\right)^{\mathrm{T}}$, and consequently the eigenvalue can be given as $\lambda=\langle A, Q\rangle$.

Lemma 3.2. Suppose that in (1.1) that the matrix $Q$ is given by $Q=\vec{s}^{\perp}\left(\vec{s}^{\perp}\right)^{\mathrm{T}}$. The eigenvalue for (1.2) is given by $\lambda=\langle A, Q\rangle$, with eigenvector equal to any multiple of $\vec{s}^{\perp}$.

This lemma is true for any $n$, and when $n=2$ we may easily compute the eigenvalue for the case $S=\operatorname{span}\{\vec{s}\}$, where

$$
\vec{s}=\left(\begin{array}{c}
\cos \theta \\
\sin \theta
\end{array}\right)
$$

This necessarily implies that $S^{\perp}=\operatorname{span}\left\{\vec{s}^{\perp}\right\}$, where

$$
\vec{s}^{\perp}=\left(\begin{array}{r}
-\sin \theta \\
\cos \theta
\end{array}\right) \Longrightarrow Q=\vec{s}^{\perp}\left(\vec{s}^{\perp}\right)^{\mathrm{T}}=\left(\begin{array}{cc}
\sin ^{2} \theta & -\cos \theta \sin \theta \\
-\cos \theta \sin \theta & \cos ^{2} \theta
\end{array}\right) .
$$

If

$$
A=\left(\begin{array}{ll}
a & b \\
c & d
\end{array}\right)
$$

then by Lemma 3.2 the eigenvalue is given by

$$
\lambda=\langle A, Q\rangle=a \sin ^{2} \theta+d \cos ^{2} \theta-(b+c) \cos \theta \sin \theta .
$$

Thus, in the case of $n=2$ the eigenvalue may always be explicitly found and is always real (assuming that the values in our matrix $A$ are real). 


\section{The EIGENVAlue PROBlem: $n=3$}

\subsection{Reformulation of the eigenvalue problem for $n \geq 3$}

First let $n \geq 3$ be arbitrary, and suppose that $S \subset \mathbb{R}^{n}$ is a subspace of dimension $k$. Write $S=$ $\operatorname{span}\left\{\vec{s}_{1}, \vec{s}_{2}, \ldots, \vec{s}_{k}\right\}$, where $\left\{\vec{s}_{1}, \vec{s}_{2}, \ldots \vec{s}_{k}\right\}$ is an orthonormal set. Any vector $\vec{v} \in \mathbb{R}^{n}$ can be written as $\vec{v}=\vec{s}+\vec{s}^{\perp}$, where $\vec{s} \in S, \vec{s}^{\perp} \in S^{\perp}$, with

$$
\vec{s}=\sum_{i=1}^{k}\left(\vec{v} \cdot \vec{s}_{i}\right) \vec{s}_{i}, \quad \vec{s}^{\perp}=\vec{v}-\vec{s} .
$$

Setting $P \vec{v}=\sum_{i=1}^{k}\left(\vec{v} \cdot \vec{s}_{i}\right) \vec{s}_{i}$ to be the projection of $\vec{v}$ onto $S$, and $Q \vec{v}=\vec{v}-P \vec{v}$ to be the projection onto $S^{\perp}$, we can note that $P \vec{s}=\vec{s}$ for $\vec{s} \in S$ and $Q \vec{s}^{\perp}=\vec{s}^{\perp}$ for $\vec{s}^{\perp} \in S^{\perp}$. Note that $k=1$ implies $P=\vec{s}_{1} \vec{s}_{1}^{T}, k=2$ implies $P=\left(\begin{array}{ll}\vec{s}_{1} & \vec{s}_{2}\end{array}\right)\left(\vec{s}_{1} \vec{s}_{2}\right)^{\mathrm{T}}$, and so on.

The following idea can be found in $[2,8]$. If $\vec{v}=\vec{s}^{\perp}$, then our eigenvalue problem (1.1) is of the form $Q A \vec{s}^{\perp}=\lambda \vec{s}^{\perp}$, because $Q \vec{s}^{\perp}=\vec{s}^{\perp}$. Now,

$$
Q A \vec{s}^{\perp}=Q\left(A \vec{s}^{\perp}\right)=A \vec{s}^{\perp}-P\left(A \vec{s}^{\perp}\right)=A \vec{s}^{\perp}-\sum_{i=1}^{k}\left[\left(A \vec{s}^{\perp}\right) \cdot \vec{s}_{i}\right] \vec{s}_{i}
$$

Letting $c_{i}=\left[\left(A \vec{s}^{\perp}\right) \cdot \vec{s}_{i}\right]$ for $i=1, \ldots, k$ then gives

$$
Q A \vec{s}^{\perp}=A \vec{s}^{\perp}-\sum_{i=1}^{k} c_{i} \vec{s}_{i}
$$

The eigenvalue problem $Q A \vec{s}^{\perp}=\lambda \vec{s}^{\perp}$ is then given by

$$
A \vec{s}^{\perp}-\sum_{i=1}^{k} c_{i} \vec{s}_{i}=\lambda \vec{s}^{\perp} \quad \Longrightarrow \quad\left(A-\lambda I_{n}\right) \vec{s}^{\perp}=\sum_{i=1}^{k} c_{i} \vec{s}_{i} .
$$

Before going further, it must be noted that if $\lambda$ is an eigenvalue of not only $Q A$ but also our matrix $A$, then $\left(A-\lambda I_{n}\right)$ is not invertible. This affects our later analysis in Section 4.2. However, this is not a problem in the formulation, for in this case the system can be solved by setting $c_{i}=0$ for $i=1, \ldots, k$. Assuming that $\lambda$ is not an eigenvalue, we invert to get

$$
\vec{s}^{\perp}=\left(A-\lambda I_{n}\right)^{-1}\left[\sum_{i=1}^{k} c_{i} \vec{s}_{i}\right]=\sum_{i=1}^{k} c_{i}\left(A-\lambda I_{n}\right)^{-1} \vec{s}_{i} .
$$

Now suppose that $k=1$, so that $S=\operatorname{span}\left\{\vec{s}_{1}\right\}$ and $\operatorname{dim}\left(S^{\perp}\right)=n-1$. From the above equation we have

$$
\vec{s}^{\perp}=c_{1}\left(A-\lambda I_{n}\right)^{-1} \vec{s}_{1} .
$$

In order for the above to be true it must be the case that $c_{1}\left(A-\lambda I_{n}\right)^{-1} \vec{s}_{1} \in S^{\perp}$, which is true if and only if this vector is orthogonal to $S$. Thus, the dot product with $\vec{s}_{1}$ must be 0 , so that

$$
c_{1}\left(A-\lambda I_{n}\right)^{-1} \vec{s}_{1} \cdot \vec{s}_{1}=0 .
$$

Set

$$
E(\lambda)=\left(A-\lambda I_{n}\right)^{-1} \vec{s}_{1} \cdot \vec{s}_{1} .
$$

The eigenvalues of $Q A Q$ in the case that $k=1$ are solutions to the secular equation $E(\lambda)=0$. Note that if we use Theorem 2.1, then we can rewrite $E(\lambda)$ as

$$
E(\lambda)=\left\langle\left(A-\lambda I_{n}\right)^{-1}, P\right\rangle, \quad P=\vec{s}_{1} \vec{s}_{1}^{T} .
$$


Theorem 4.1. Let $A$ be an $n \times n$ matrix, let $P=\vec{s} \vec{s}^{T}$ be the projection matrix onto $S=\operatorname{span}\{\vec{s}\}$, and let $Q=I_{n}-P$ be the projection matrix onto $S^{\perp}$, the $(n-1)$-dimensional space perpendicular to $S$. Set

$$
E(\lambda)=\left\langle\left(A-\lambda I_{n}\right)^{-1}, P\right\rangle .
$$

The eigenvalues for problem (1.2) are realized either as

- the zeros of the function, or

- a removable singularity of $E(\lambda)$.

Remark 4.2. The poles of the rational function $E(\lambda)$ are realized as the eigenvalues of the matrix $A$. The standard theory associated with rational functions reveals that near a simple pole $\lambda_{0}$ the function $E(\lambda)$ will have associated with it the Laurent series

$$
E(\lambda)=\frac{r}{\lambda-\lambda_{0}}+\sum_{j=0}^{\infty} e_{j}\left(\lambda-\lambda_{0}\right)^{j}
$$

[11]. The number $r$ is known as the residue, and the singularity is said to be removable if $r=0$. If $\lambda_{0}$ is an eigenvalue of $A$, then the expression $\left(A-\lambda_{0} I_{n}\right)^{-1} \vec{s}$ for $\vec{s} \in S$ may make sense in the following manner. Suppose that $A$ is symmetric. By the Fredholm alternative the system

$$
\left(A-\lambda_{0} I\right) \vec{x}=\vec{s}, \quad \vec{s} \in S
$$

has a unique solution $\vec{x} \in S^{\perp}$ (compare with (4.1)) if $\vec{s} \cdot \vec{a}=0$, where $A \vec{a}=\lambda_{0} \vec{a}$. Thus, if for a given eigenvalue $\lambda_{0}$ with associated eigenvector $\vec{a}$ it is true that $\vec{a} \in S^{\perp}$, then by the construction of $E(\lambda)$ the function will have a removable singularity at $\lambda_{0}$.

Now suppose that $A=B D B^{-1}$. Since

$$
A-\lambda I_{n}=B\left(D-\lambda I_{n}\right) B^{-1} \quad \Longrightarrow \quad\left(A-\lambda I_{n}\right)^{-1}=B\left(D-\lambda I_{n}\right)^{-1} B^{-1},
$$

we can rewrite $E(\lambda)$ as

$$
E(\lambda)=B\left(D-\lambda I_{n}\right)^{-1} B^{-1} \vec{s} \cdot \vec{s}=\left(D-\lambda I_{n}\right)^{-1} B^{-1} \vec{s} \cdot B^{\mathrm{T}} \vec{s} .
$$

Using the remark after Theorem 2.1 then gives us that

$$
E(\lambda)=\left\langle\left(D-\lambda I_{n}\right)^{-1},\left(B^{\mathrm{T}} \vec{s}\right)\left(B^{-1} \vec{s}\right)^{\mathrm{T}}\right\rangle .
$$

Let $\vec{x}=B^{\mathrm{T}} \vec{s}$ and $\vec{y}=B^{-1} \vec{s}$. Since $\vec{s}=B \vec{y}$, it is then true that $\vec{x}=B^{\mathrm{T}} B \vec{y}$, so

$$
E(\lambda)=\left\langle\left(D-\lambda I_{n}\right)^{-1}, \vec{x} \vec{y}^{\mathrm{T}}\right\rangle=\left\langle\left(D-\lambda I_{n}\right)^{-1},\left(B^{\mathrm{T}} B \vec{y}\right) \vec{y}^{\mathrm{T}}\right\rangle .
$$

The matrix $H=B^{\mathrm{T}} B$ is symmetric, so one can finally rewrite $E(\lambda)$ as

$$
E(\lambda)=\left\langle\left(D-\lambda I_{n}\right)^{-1},(H \vec{y}) \vec{y}^{\mathrm{T}}, \quad H=H^{\mathrm{T}}\right\rangle .
$$

Corollary 4.3. In Theorem 4.1 suppose that $A=B D B^{-1}$. Then for

$$
H=B^{\mathrm{T}} B, \quad \vec{y}=B^{-1} \vec{s},
$$

we have

$$
E(\lambda)=\left\langle\left(D-\lambda I_{n}\right)^{-1},(H \vec{y}) \vec{y}^{\mathrm{T}}\right\rangle
$$




\subsection{Case study: $n=3$}

We will now focus on the case that $n=3$. We will use the formulation of $E(\lambda)$ in Corollary 4.3 to study the eigenvalue problem (1.1), with $A=B D B^{-1}$, so that $D$ will be quasi-upper triangular. Note that this diagonalizes $A$, with the columns of matrix $B$ being (generalized) eigenvectors and matrix $D$ is in Jordan canonical form, allowing us to identify the four different canonical diagonalized cases for the matrix $D$. We will primarily focus on the case when $H=I_{3}$, so that $(H \vec{y}) \vec{y}^{\mathrm{T}}=P$, where $P$ is given in Theorem 4.1. In particular,

$$
\vec{s}=\left(\begin{array}{c}
p \\
q \\
r
\end{array}\right) \quad \Longrightarrow \quad P=\left(\begin{array}{ccc}
p^{2} & p q & p r \\
p q & q^{2} & q r \\
p r & q r & r^{2}
\end{array}\right)
$$

with $p^{2}+q^{2}+r^{2}=1$. We will conclude by looking again at Case 1 for a special case of $H \neq I_{3}$.

$$
\text { 4.2.1. Case 1: } A=D=\left(\begin{array}{ccc}
\lambda_{1} & 0 & 0 \\
0 & \lambda_{2} & 0 \\
0 & 0 & \lambda_{3}
\end{array}\right)
$$

In this case

$$
E(\lambda)=\left\langle\left(\begin{array}{ccc}
\frac{1}{\left(\lambda_{1}-\lambda\right)} & 0 & 0 \\
0 & \frac{1}{\left(\lambda_{2}-\lambda\right)} & 0 \\
0 & 0 & \frac{1}{\left(\lambda_{3}-\lambda\right)}
\end{array}\right), P\right\rangle=\frac{p^{2}}{\left(\lambda_{1}-\lambda\right)}+\frac{q^{2}}{\left(\lambda_{2}-\lambda\right)}+\frac{r^{2}}{\left(\lambda_{3}-\lambda\right)}
$$

From this formulation we can see that the positive/negative values of our elements of $\vec{s}$ do not matter, as they are squared. There are vertical asymptotes at $\lambda_{1}, \lambda_{2}, \lambda_{3}$, and because our numerators are always positive, then the denominators control the signs of each term, and thus where the zeroes exist. We can state $\lambda_{1}<\lambda_{2}<\lambda_{3}$ without loss of generality, thus allowing us to note that the graph follows the form of Figure 1. Thus our zeros are always between our asymptotes, or with our generalization, our zeroes exist in the regions $\lambda_{1}<\lambda<\lambda_{2}$ and $\lambda_{2}<\lambda<\lambda_{3}$ (see Appendix for full analysis). We should also note that these results follow from the Cauchy Interlacing Theorem.

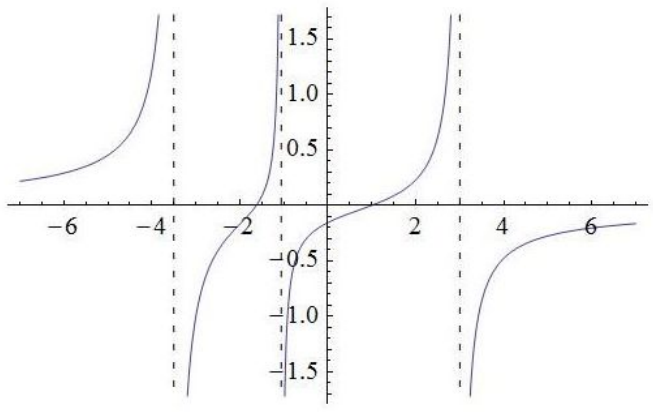

Figure 1: (color online) A plot of $E(\lambda)$ for Case 1 when $\lambda_{1}<\lambda_{2}<\lambda_{3}$ and $H=I_{3}$. 
4.2.2. Case 2: $A=D=\left(\begin{array}{ccc}\lambda_{1} & 1 & 0 \\ 0 & \lambda_{1} & 0 \\ 0 & 0 & \lambda_{2}\end{array}\right)$

In this case

$$
E(\lambda)=\left\langle\left(\begin{array}{ccc}
\frac{1}{\left(\lambda_{1}-\lambda\right)} & -\frac{1}{\left(\lambda_{1}-\lambda\right)^{2}} & 0 \\
0 & \frac{1}{\left(\lambda_{1}-\lambda\right)} & 0 \\
0 & 0 & \frac{1}{\left(\lambda_{2}-\lambda\right)}
\end{array}\right), P\right\rangle=\frac{p^{2}+q^{2}}{\left(\lambda_{1}-\lambda\right)}-\frac{p q}{\left(\lambda_{1}-\lambda\right)^{2}}+\frac{r^{2}}{\left(\lambda_{2}-\lambda\right)} .
$$

As seen in Figure 2, the plot of $E(\lambda)$ has four possible general forms (full analysis in Appendix). The zeroes are dependent upon whether $p q<0$ or $p q>0$, and whether $\lambda_{1}>\lambda_{2}$ or $\lambda_{1}<\lambda_{2}$. It is possible for complex roots to exist in this canonical case, e.g., the graph of the function in the case of $p q<0$ and $\lambda_{1}>\lambda_{2}$ may be completely above the x-axis, thus causing $E(\lambda)$ to have no real roots.

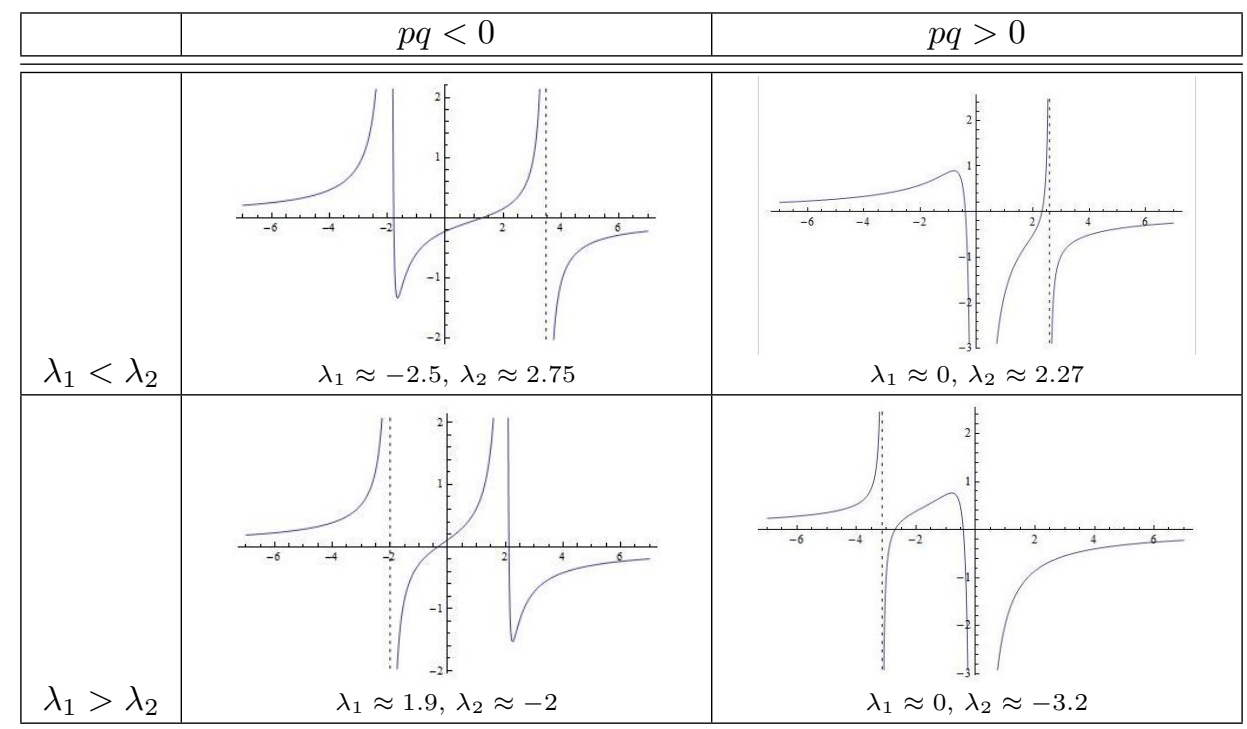

Figure 2: (color online) A plot of $E(\lambda)$ for Case 2 when $H=I_{3}$. Although we have not demonstrated it here, it is possible in the top left panel and bottom right panel for $E(\lambda)=0$ to have no real-valued solutions.

4.2.3. Case 3: $A=D=\left(\begin{array}{ccc}\lambda_{1} & 1 & 0 \\ 0 & \lambda_{1} & 1 \\ 0 & 0 & \lambda_{1}\end{array}\right)$

In this case

$$
\begin{aligned}
E(\lambda) & \left.=\left\langle\begin{array}{ccc}
\frac{1}{\left(\lambda_{1}-\lambda\right)} & -\frac{1}{\left(\lambda_{1}-\lambda\right)^{2}} & \frac{1}{\left(\lambda_{1}-\lambda\right)^{3}} \\
0 & \frac{1}{\left(\lambda_{1}-\lambda\right)} & \frac{-1}{\left(\lambda_{1}-\lambda\right)^{2}} \\
0 & 0 & \frac{1}{\left(\lambda_{1}-\lambda\right)}
\end{array}\right), P\right\rangle \\
& =\frac{p^{2}}{\left(\lambda_{1}-\lambda\right)}-\frac{p q}{\left(\lambda_{1}-\lambda\right)^{2}}+\frac{p r}{\left(\lambda_{1}-\lambda\right)^{3}}+\frac{q^{2}}{\left(\lambda_{1}-\lambda\right)}-\frac{q r}{\left(\lambda_{1}-\lambda\right)^{2}}+\frac{r^{2}}{\left(\lambda_{1}-\lambda\right)}
\end{aligned}
$$


Upon using the fact that $p^{2}+q^{2}+r^{2}=1$, this expression can be reduced to

$$
E(\lambda)=\frac{1}{\left(\lambda_{1}-\lambda\right)}-\frac{p q+q r}{\left(\lambda_{1}-\lambda\right)^{2}}+\frac{p r}{\left(\lambda_{1}-\lambda\right)^{3}} .
$$

This canonical case has three different general forms for a graph (see Figure 3), dependent upon whether $p r>0$ or $p r<0$ as well as the sign of $q$ with respect to $p$ and $r$ (this case only changes the general form of the graph if $p r>0$, full analysis in Appendix). We do know for a fact that if $p r<0$, then there exist two real roots, one in each of the regions $\lambda>\lambda_{1}$ and $\lambda<\lambda_{1}$. Otherwise, it is entirely possible that two imaginary roots exist.

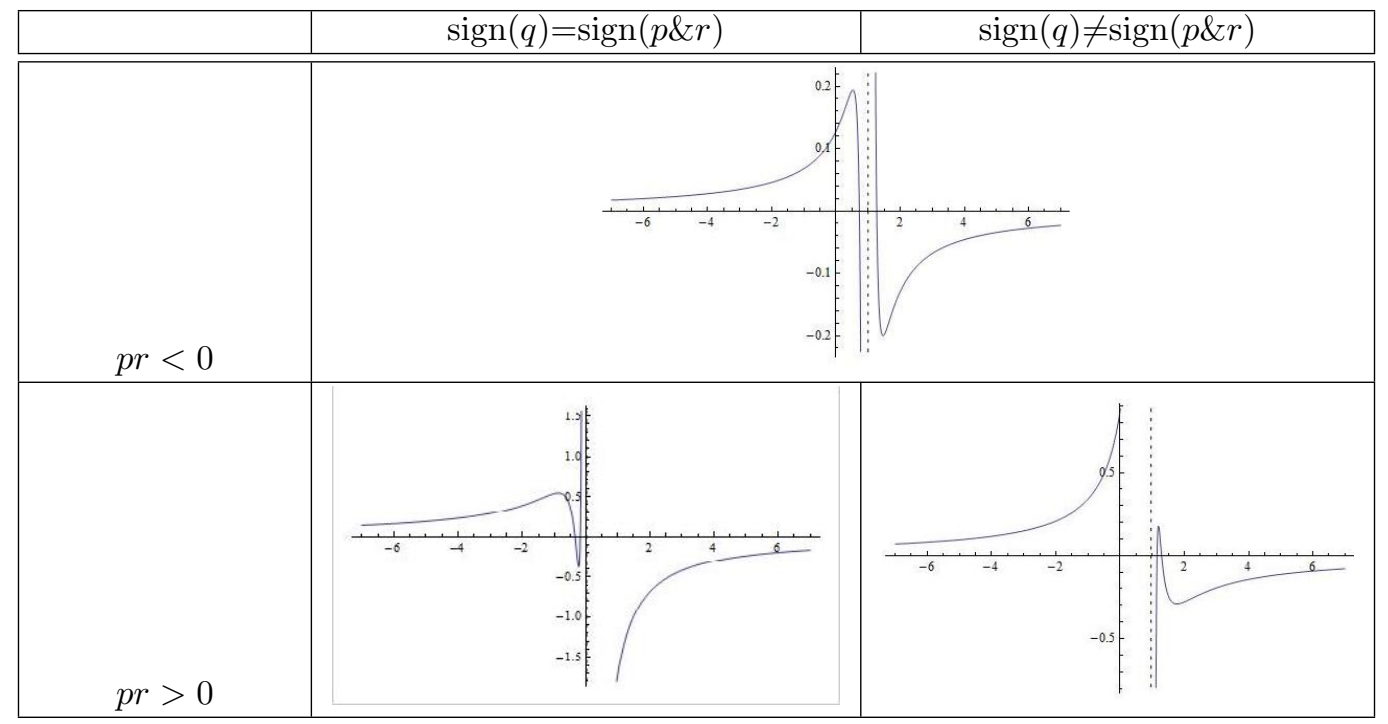

Figure 3: (color online) A plot of $E(\lambda)$ for Case 3 when $H=I_{3}$. Although we have not demonstrated it here, it is possible in the bottom two panels for $E(\lambda)=0$ to have no real-valued solutions.

4.2.4. Case 4: $A=D=\left(\begin{array}{rrr}\lambda_{1} & 0 & 0 \\ 0 & a & b \\ 0 & -b & a\end{array}\right)$

In this case

$$
\begin{aligned}
E(\lambda) & \left.=\left\langle\begin{array}{ccc}
\frac{1}{\left(\lambda_{1}-\lambda\right)} & 0 & 0 \\
0 & \frac{(a-\lambda)}{(a-\lambda)^{2}+b^{2}} & -\frac{b}{(a-\lambda)^{2}+b^{2}} \\
0 & \frac{b}{(a-\lambda)^{2}+b^{2}} & \frac{(a-\lambda)}{(a-\lambda)^{2}+b^{2}}
\end{array}\right), P\right\rangle \\
& =\frac{p^{2}}{\left(\lambda_{1}-\lambda\right)}+\frac{\left(q^{2}+r^{2}\right)(a-\lambda)}{(a-\lambda)^{2}+b^{2}} .
\end{aligned}
$$

Using the fact that $p^{2}+q^{2}+r^{2}=1$, we can rewrite the above as

$$
E(\lambda)=\frac{p^{2}}{\left(\lambda_{1}-\lambda\right)}+\frac{\left(1-p^{2}\right)(a-\lambda)}{(a-\lambda)^{2}+b^{2}} .
$$

This case only has two general forms for graphs, dependent upon whether $a>\lambda_{1}$ or $a<\lambda_{1}$ (see Figure 4). Definitive conclusions (see Appendix for details) include that there could exist two imaginary roots, but if 
two real roots exist then they must be either both less than $\lambda_{1}$, or both greater. Also of note is the fact that in the case that

$$
0>\lambda_{1} E(0)=p^{2}+\frac{\left(1-p^{2}\right) a \lambda_{1}}{a^{2}+b^{2}},
$$

then we know that there exist two real roots, one positive and one negative.

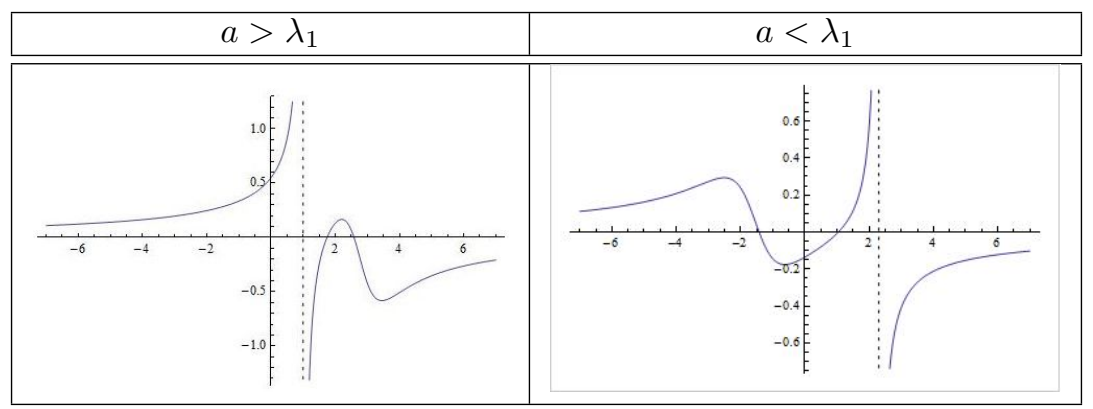

Figure 4: (color online) A plot of $E(\lambda)$ for Case 4 when $H=I_{3}$. Note that in the left panel $\lambda_{1} E(0)>0$, so that it is possible to have no real-valued solutions to $E(\lambda)=0$, while in the right panel $\lambda_{1} E(0)<0$, so that there will necessarily be two real-valued solutions.

\subsubsection{Case $1, H \neq I_{3}$}

Suppose that $D$ is given as in Case I, and further suppose that

$$
B=\left(\begin{array}{ccc}
b_{1} & b_{2} & 0 \\
b_{3} & b_{4} & 0 \\
0 & 0 & b_{5}
\end{array}\right) \quad \Longrightarrow \quad H=\left(\begin{array}{ccc}
b_{1}^{2}+b_{2}^{2} & b_{1} b_{3}+b_{2} b_{4} & 0 \\
b_{1} b_{3}+b_{2} b_{4} & b_{3}^{2}+b_{4}^{2} & 0 \\
0 & 0 & b_{5}^{2}
\end{array}\right)
$$

Set

$$
a=b_{1}^{2}+b_{2}^{2}, b=b_{1} b_{3}+b_{2} b_{4}, d=b_{3}^{2}+b_{4}^{2}, f=b_{5}^{2},
$$

and note that $a, f, a d-b^{2}>0$, so that $H$ is a positive-definite symmetric matrix (note also that since $H=B^{\mathrm{T}} B$ for a real, invertible $B, H$ will always be symmetric and positive-definite, i.e., all of the eigenvalues of $H$ will be positive). Letting $\vec{y}=\left(y_{1}, y_{2}, y_{3}\right)^{\mathrm{T}}$ then yields from Corollary 4.3 that

$$
E(\lambda)=a \frac{y_{1}^{2}}{\left(\lambda_{1}-\lambda\right)}+d \frac{y_{2}^{2}}{\left(\lambda_{2}-\lambda\right)}+f \frac{y_{3}^{2}}{\left(\lambda_{3}-\lambda\right)}+b y_{1} y_{2}\left(\frac{1}{\left(\lambda_{1}-\lambda\right)}+\frac{1}{\left(\lambda_{2}-\lambda\right)}\right) .
$$

This equation is similar to the equation presented in Case I, except for the existence of $b$. In particular, if $b=0$ and $a, d, f>0$, then there is no difference in the conclusion. However, it is possible to choose values for $a, b, d$, and $f$ such that no real roots exist, thus breaking the definitive result found in canonical Case 1 (see Figure 5).

Remark 4.4. Since Case 1 can be altered in this way, it is logical that the other canonical cases can also be altered via this method to break their definitive results as well. Note that this alteration in B also allows the eigenvectors to no longer be orthogonal, and thus A is no longer a normal matrix and the Cauchy interlacing result no longer applies.

\section{CONCLUSION}

We have discovered methods of finding the eigenvalues for the eigenvalue problem (1.1) in the case that $\operatorname{dim}(S)=1$. We examined in some detail the problem when $n=2,3$. As of yet, there are still cases where we 


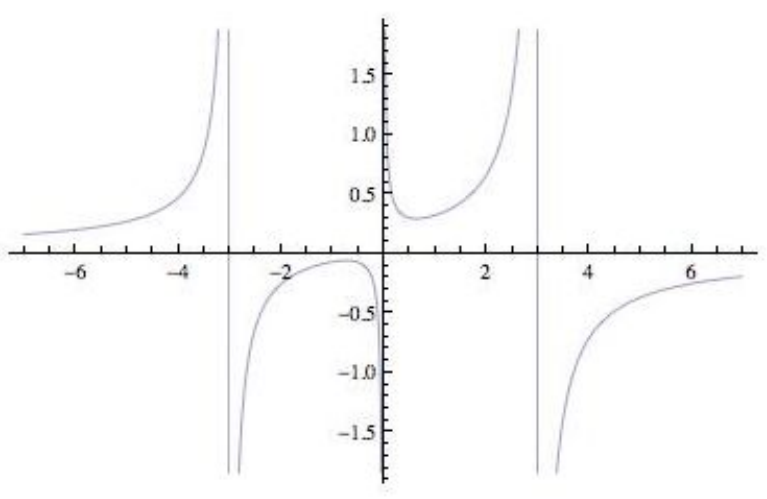

Figure 5: A plot of $E(\lambda)$ for Case $1, H \neq I_{3}$, when $\left(\lambda_{1}, \lambda_{2}, \lambda_{3}\right)=(-3,0,3),\left(y_{1}, y_{2}, y_{3}\right)=$ $(1 / 2,1 / 4,3 / 4), a=2$, and $d=f=b=1$.

do not know a simple technique of analyzing in detail the problem for $n \geq 4$ and/or $\operatorname{dim}(S) \geq 2$. However, this research is a beginning towards understanding these problems, and perhaps future research will reveal these still-hidden methods.

\section{APPENDIX}

\section{Case 1}

Here

$$
E(\lambda)=\frac{p^{2}}{\left(\lambda_{1}-\lambda\right)}+\frac{q^{2}}{\left(\lambda_{2}-\lambda\right)}+\frac{r^{2}}{\left(\lambda_{3}-\lambda\right)} .
$$

Recall that we are assuming $\lambda_{1}<\lambda_{2}<\lambda_{3}$. It is clear that asymptotes exist at $\lambda_{1}, \lambda_{2}, \lambda_{3}$. Since the numerators are positive, we also have

$$
\lim _{\lambda \rightarrow \lambda_{j}^{ \pm}} E(\lambda)=\mp \infty, \quad j=1, \ldots, 3 .
$$

It is clear that

$$
\lim _{\lambda \rightarrow \pm \infty} E(\lambda)=0
$$

If $-\infty<\lambda<\lambda_{1}$, then $\left(\lambda_{j}-\lambda\right)>0$ for $j=1, \ldots, 3$ implies that $E(\lambda)>0$, and if $\lambda_{3}<\lambda<\infty$, then $\left(\lambda_{j}-\lambda\right)<0$ for $j=1, \ldots, 3$, so that $E(\lambda)<0$. Finally,

$$
E^{\prime}(\lambda)=\frac{p^{2}}{\left(\lambda_{1}-\lambda\right)^{2}}+\frac{q^{2}}{\left(\lambda_{2}-\lambda\right)^{2}}+\frac{r^{2}}{\left(\lambda_{3}-\lambda\right)^{2}}>0,
$$

so that the function is always increasing. This analysis produces the graph of Figure 1. Since the only asymptotes are at $\lambda_{1}, \lambda_{2}, \lambda_{3}$, then $E(\lambda)$ moves from $-\infty$ to $\infty$ in the intervals $\lambda_{1}<\lambda<\lambda_{2}$ and $\lambda_{2}<\lambda<\lambda_{3}$; thus, the zeros are in these same intervals.

\section{Case 2}

Here

$$
E(\lambda)=\frac{p^{2}+q^{2}}{\left(\lambda_{1}-\lambda\right)}-\frac{p q}{\left(\lambda_{1}-\lambda\right)^{2}}+\frac{r^{2}}{\left(\lambda_{2}-\lambda\right)} .
$$


Asymptotes clearly exist at $\lambda_{1}$ and $\lambda_{2}$, with

$$
\lim _{\lambda \rightarrow \lambda_{2}^{ \pm}} E(\lambda)=\mp \infty
$$

Regarding the behavior for large real $\lambda$ we have

$$
\lim _{\lambda \rightarrow \pm \infty} E(\lambda)=0
$$

furthermore, $E(\lambda)>0$ for $\lambda \ll-1$, and $E(\lambda)<0$ for $\lambda \gg 1$, because for large $|\lambda|$ the dominant terms in the sum have positive numerators.

$$
\text { Case 2-a: } \lambda_{1}<\lambda_{2} \text { and } p q<0
$$

We have

$$
\lim _{\lambda \rightarrow \lambda_{1}^{ \pm}} E(\lambda)=+\infty
$$

since the dominant term in the sum for $\lambda$ near $\lambda_{1}$ is $-p q /\left(\lambda_{1}-\lambda\right)^{2}>0$. This analysis yields the conclusion that if there do exist real zeros, then they exist in the interval $\lambda_{1}<\lambda<\lambda_{2}$.

\section{Case 2-b: $\lambda_{1}<\lambda_{2}$ and $p q>0$}

We have

$$
\lim _{\lambda \rightarrow \lambda_{1}^{ \pm}} E(\lambda)=-\infty
$$

since the dominant term in the sum for $\lambda$ near $\lambda_{1}$ is $-p q /\left(\lambda_{1}-\lambda\right)^{2}<0$. Since $E(\lambda)>0$ for $\lambda \ll-1$, by continuity it must then be true that $E(\lambda)=0$ has at least one real-valued solution in the interval $-\infty<\lambda<\lambda_{1}$. Furthermore, because of continuity another zero exists in the interval $\lambda_{1}<\lambda<\lambda_{2}$.

$$
\text { Case 2-c: } \lambda_{1}>\lambda_{2} \text { and } p q<0
$$

As in Case 2-a we have

$$
\lim _{\lambda \rightarrow \lambda_{1}^{ \pm}} E(\lambda)=+\infty
$$

Using the same analysis as for Case 2-b, this then implies that $E(\lambda)=0$ has at least one solution in the interval $\lambda_{2}<\lambda<\lambda_{1}$. Since $E(\lambda)<0$ for $\lambda \gg 1$, this implies that $E(\lambda)=0$ has at least one solution in the interval $\lambda_{1}<\lambda<+\infty$.

Case 2-d: $\lambda_{1}>\lambda_{2}$ and $p q>0$

As in Case 2-b we have

$$
\lim _{\lambda \rightarrow \lambda_{1}^{ \pm}} E(\lambda)=-\infty
$$

Using the same analysis as for Case 2-a, we can conclude that any real zeros for $E(\lambda)$ must occur in the interval $\lambda_{2}<\lambda<\lambda_{1}$. Case 2-c and 2-d may also be seen to follow from 2-a and 2-b by replacing $A$ with $-A$. 


\section{Case 3}

Here

$$
E(\lambda)=\frac{1}{\left(\lambda_{1}-\lambda\right)}-\frac{p q+q r}{\left(\lambda_{1}-\lambda\right)^{2}}+\frac{p r}{\left(\lambda_{1}-\lambda\right)^{3}} .
$$

We have that

$$
\lim _{\lambda \rightarrow \pm \infty} E(\lambda)=0
$$

furthermore, for large $|\lambda|$ the first term in the sum is dominant, so that $E(\lambda)>0$ for $\lambda \ll-1$ and $E(\lambda)<0$ for $\lambda \gg 1$.

An asymptote exists only at $\lambda=\lambda_{1}$.

\section{Case 3-a: $p r<0$}

For $\lambda$ near $\lambda_{1}$ the third term in the sum will be dominant; hence,

$$
\lim _{\lambda \rightarrow \lambda_{1}^{ \pm}} E(\lambda)= \pm \infty .
$$

Because $E(\lambda)$ is continuous there must then exist at least one zero in each of the intervals $\left(-\infty, \lambda_{1}\right)$ and $\left(\lambda_{1}, \infty\right)$.

Case 3-b: $p r>0$

For $\lambda$ near $\lambda_{1}$ the third term in the sum will be dominant; hence,

$$
\lim _{\lambda \rightarrow \lambda_{1}^{ \pm}} E(\lambda)=\mp \infty .
$$

Now if $\operatorname{sign}(q)=\operatorname{sign}(p \& r)(p$ and $r$ must have the same sign since $p r>0)$, then $p q>0$ and $p r>0$, which implies that $p q+q r>0$. Then in the interval $\lambda_{1}<\lambda<\infty$ each term in the sum will be negative, so that $E(\lambda)<0$. Thus, if real zeros exist, they must exist in the interval $-\infty<\lambda<\lambda_{1}$.

If $\operatorname{sign}(q) \neq \operatorname{sign}(p \& r)$, then $p q<0$ and $p r<0$, which implies that $p q+q r<0$. Then in the interval $-\infty<\lambda<\lambda_{1}$ each term in the sum will be positive, implying that $E(\lambda)>0$ in this interval. Thus if real zeros exist, they must exist in the interval $\lambda_{1}<\lambda<\infty$.

\section{Case 4}

Here

$$
E(\lambda)=\frac{p^{2}}{\left(\lambda_{1}-\lambda\right)}+\frac{\left(1-p^{2}\right)(a-\lambda)}{(a-\lambda)^{2}+b^{2}} .
$$

An asymptote exists only at $\lambda=\lambda_{1}$, with

$$
\lim _{\lambda \rightarrow \lambda_{1}^{ \pm}} E(\lambda)=\mp \infty
$$

In addition, we have that

$$
\lim _{\lambda \rightarrow \pm \infty} E(\lambda)=0
$$

furthermore, since $p^{2} \leq 1$ we have that $E(\lambda)>0$ for $\lambda \ll-1$ and $E(\lambda)<0$ for $\lambda \gg 1$.

\section{Case 4-a: $a>\lambda_{1}$}

In this case each term in the sum, and hence $E(\lambda)$ itself, is positive for $\lambda<\lambda_{1}$. Thus, if zeros exist then they must exist in the interval $\lambda_{1}<\lambda<\infty$. 


\section{Case 4-b: $a<\lambda_{1}$}

In this case each term in the sum, and hence $E(\lambda)$ itself, is negative for $\lambda>\lambda_{1}$. Thus, if zeros exist then they must exist in the interval $-\infty<\lambda<\lambda_{1}$.

$$
\text { Case 4-c: } \lambda_{1} E(0)<0
$$

We also note that if $\lambda_{1} E(0)<0$ then some conclusions may be drawn. Since

$$
\lambda_{1} E(0)=\frac{p^{2}\left(a^{2}+b^{2}\right)+\left(1-p^{2}\right) \lambda_{1} a}{\left(a^{2}+b^{2}\right)},
$$

we have that $\lambda_{1} E(0)<0$ if and only if

$$
\lambda_{1} a<-\frac{p^{2}}{1-p^{2}}\left(a^{2}+b^{2}\right)<0 .
$$

If $\lambda_{1}<0$ and $E(0)>0$, then there exist an odd number of zeros in the interval $\lambda_{1}<\lambda<0$. Since $E(\lambda)<0$ for large positive $\lambda$, there also exist an odd number of zeros in the interval $0<\lambda<+\infty$. Since there are only two zeros, there is then precisely one zero in the interval $\lambda_{1}<\lambda<0$, and one zero in the interval $0<\lambda<+\infty$. On the other hand, if $\lambda_{1}>0$ and $E(0)<0$, then applying the same argument gives one zero in the interval $-\infty<\lambda<0$, and another zero in the interval $0<\lambda<\lambda_{1}$. In conclusion, in this case there will always be two real-valued zeros.

\section{REFERENCES}

[1] R. Courant and D. Hilbert. Methods of Mathematical Physics, volume 1. Interscience Publishers, Inc., 1953.

[2] S. Cuccagna, D. Pelinovsky, and V. Vougalter. Spectra of positive and negative energies in the linearized NLS problem. Comm. Pure Appl. Math., 58:1-29, 2005.

[3] B. Deconinck and T. Kapitula. The orbital stability of the cnoidal waves of the Korteweg-de Vries equation. Phys. Letters A, 374:4018-4022, 2010.

[4] J. Hefferon. Linear Algebra. 2011. http://joshua.smcvt.edu/linalg.html/.

[5] H. Hochstadt. On the construction of a Jacobi matrix from spectral data. Lin. Algebra Appl., 8:435-446, 1974.

[6] T. Kapitula. On the stability of $N$-solitons in integrable systems. Nonlinearity, 20(4):879-907, 2007.

[7] T. Kapitula. The Krein signature, Krein eigenvalues, and the Krein Oscillation Theorem. Indiana U. Math. J., 59:1245-1276, 2010.

[8] T. Kapitula and K. Promislow. Stability indices for constrained self-adjoint operators. Proc. Amer. Math. Soc., 140(3):865-880, 2012.

[9] T. Kapitula, P. Kevrekidis, and B. Sandstede. Counting eigenvalues via the Krein signature in infinitedimensional Hamiltonian systems. Physica D, 195(3\&4):263-282, 2004.

[10] S. Malamud. Inverse spectral problem for normal matrices and the Gauss-Lucas theorem. Transactions AMS, 357(10):4043-4064, 2005.

[11] J. Mathews and R. Howell. Complex Analysis for Mathematics and Engineering. Jones and Bartlett Publishers, Fifth edition, 2006. 